\title{
Supplemental oxygen during exercise training in COPD: full of hot air?
}

\author{
Jessica A. Walsh ${ }^{1}$, Matthew Maddocks ${ }^{2}$ and William D-C. Man ${ }^{1,3}$ \\ Affiliations: ${ }^{1}$ Harefield Respiratory Research Group, Royal Brompton and Harefield NHS Foundation Trust, \\ Uxbridge, UK. ${ }^{2}$ Cicely Saunders Institute of Palliative Care, Policy and Rehabilitation, King's College London, \\ London, UK. ${ }^{3}$ National Heart and Lung Institute, Imperial College London, London, UK.
}

Correspondence: William D-C. Man. Harefield Respiratory Research Group, Dept of Respiratory Medicine, Harefield Hospital, Hill End Rd, Harefield, Uxbridge UB9 6JH, UK. Email: w.mandarbht.nhs.uk

@ERSpublications

The majority of patients with COPD and exercise induced desaturation safely benefit from exercise training without the need for supplemental oxygen http://bit.ly/2Yof9xJ

Cite this article as: Walsh JA, Maddocks M, Man WD-C. Supplemental oxygen during exercise training in COPD: full of hot air? Eur Respir J 2019; 53: 1900837 [https://doi.org/10.1183/13993003.00837-2019].

Long-term oxygen therapy is widely accepted as a treatment for severe hypoxaemia in patients with chronic respiratory disorders, based on survival findings from the UK Medical Research Council study [1] and the US Nocturnal Oxygen Therapy Trial [2]. The data from these landmark studies have driven the underlying rationale that correcting hypoxaemia is beneficial, and have led to the prescription of oxygen for other indications, including ambulatory oxygen for patients with exercise-induced oxygen desaturation (EID), nocturnal oxygen therapy for patients with nocturnal hypoxaemia and short burst oxygen for palliation of dyspnoea. This is despite a lack of supporting evidence from randomised clinical trials to support these indications [3-5].

A clinical indication that stimulates long-standing debate is the specific use of supplemental oxygen during exercise training in patients with EID. There is a sound physiological rationale in that supplemental oxygen can reduce respiratory rate and dynamic hyperinflation [6]. It also improves oxygen delivery, leading to a reduction in exercise-associated metabolic acidosis [7]. More than 60 years ago, Cotes and GILSON [8] demonstrated that ambulatory oxygen could support outpatients with chronic respiratory insufficiency during exercise by improving arterial oxygen saturation and increasing walking time. Subsequent, largely laboratory-based, studies have demonstrated a positive, albeit heterogeneous, benefit in exercise time following acute administration of oxygen during an exercise test in patients with chronic obstructive pulmonary disease (COPD) or cystic fibrosis $[9,10]$. This had led to the logical hypothesis that by improving exercise time acutely, supplemental oxygen allows patients to tolerate training sessions of higher intensity with subsequent greater gains from an exercise training programme. Although there have been attempts to provide evidence to support this hypothesis [11-14], the results from clinical trials have been mixed and inconclusive, largely due to methodological issues, small sample sizes and inclusion of a heterogeneous population, including existing long-term oxygen users [11] or individuals without EID [13, 14].

In this issue of the European Respiratory Journal, Auson et al. [15] report on a double-blind randomised placebo-controlled trial that aimed to determine whether supplemental oxygen administered during exercise training was more effective than medical air in improving endurance exercise capacity, health-related quality of life, peak walking capacity, dyspnoea and physical activity levels in patients with COPD who desaturated during exercise ( $<90 \%$ during the 6 -min walk test) but were normoxaemic at rest. Following random allocation to either oxygen or compressed air, both groups underwent an 8 -week 


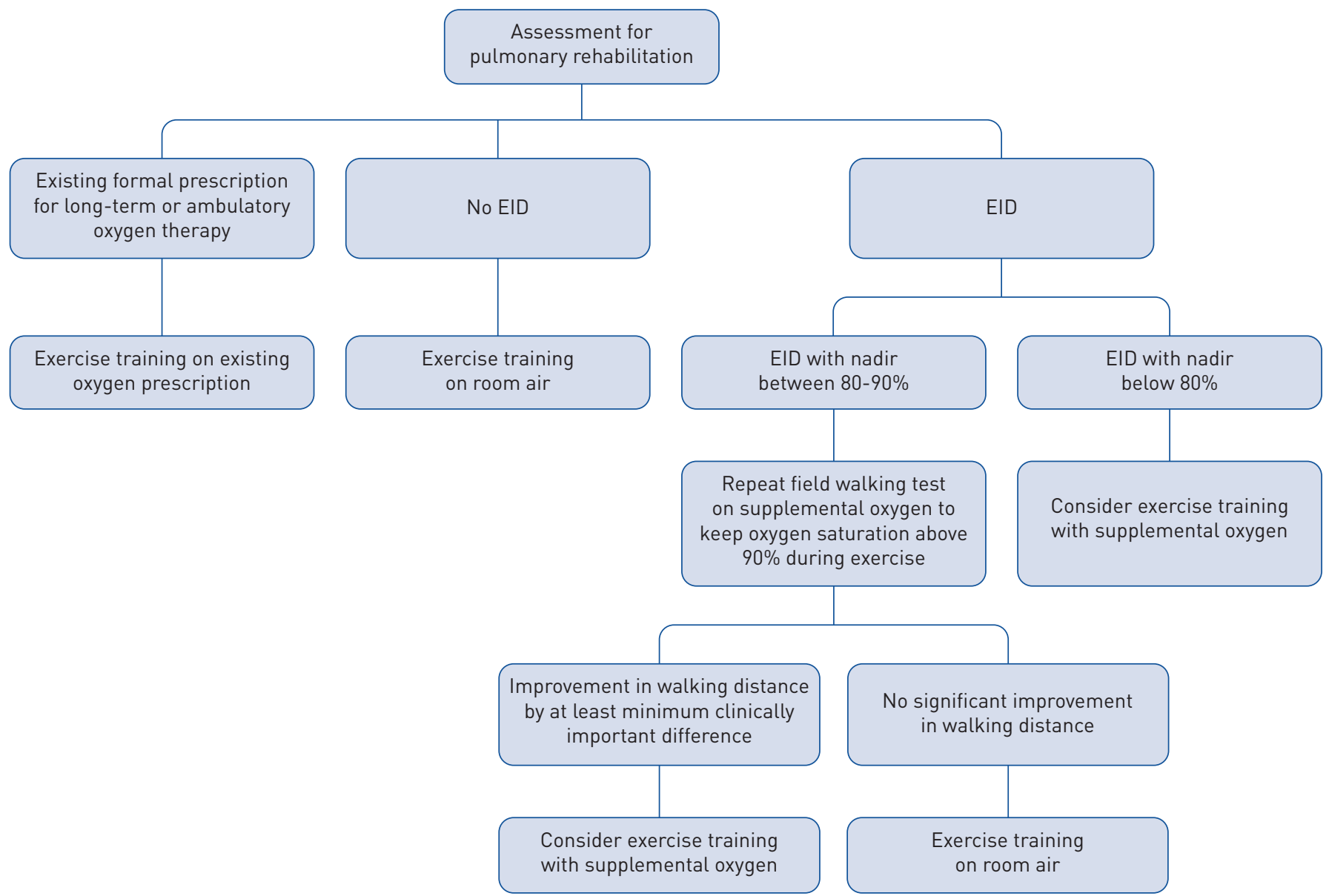

FIGURE 1 An algorithm to assess exercise induced oxygen desaturation (EID) (defined as nadir oxygen saturation below $90 \%$ during a field walking test performed on room air) in patients with chronic obstructive pulmonary disease referred for pulmonary rehabilitation.

training programme with three supervised exercise sessions per week. Training initially consisted of $30 \mathrm{~min}$ of individualised aerobic exercise (20 min of treadmill walking and $10 \mathrm{~min}$ of stationary cycling). Training intensity was increased according to target dyspnoea scores (3-4 on modified dyspnoea scale [16]) and training duration was increased according to a fixed protocol. The results of this study demonstrated that both groups improved exercise capacity and health-related quality of life following exercise training, but no added benefit was seen with supplemental oxygen in either of the primary outcomes measures of endurance shuttle walk test and Chronic Respiratory Questionnaire, nor in any of the secondary outcome measures. This was a well-designed and conducted study, with clear eligibility criteria, high retention of participants, and successful blinding of participants, trainers and assessors: a feature absent in previous trials [11-14].

A fundamental observation was that the oxygen group were not able to achieve a greater training dose per session than the compressed air group, despite a significantly higher measured oxygen saturation and significantly lower dyspnoea and perceived exertion scores during treadmill training sessions. Without an increased training load, it was not altogether surprising that no between-group differences in response to exercise-training were observed. The authors speculated that the large physiological stimulus applied to both groups overwhelmed the small physiological advantage of acute oxygen administration. Although this is a highly plausible explanation (observed in previous pulmonary rehabilitation adjunct trials $[17,18]$ ), other mechanisms may have limited exercise-training load. The authors did not provide data regarding leg effort or lower limb symptoms, which are reported frequently as a reason for terminating exercise in a proportion of patients with COPD [19]. The exercise-training programme was protocolised such that exercise type and frequency were fixed and exercise duration was progressed up to a maximum of 40 min by week three. From this point onwards, staff supervising exercise-training could only increase training intensity (speed of treadmill and cycle workload) but not duration of training: the parameter that is most consistently influenced by acute oxygen response studies. Another speculative explanation is that because the 
trainers were blinded to treatment allocation and to patients' oxygen saturation during training, they may have inadvertently been more cautious of increasing training intensity in patients known to desaturate during exercise (of whom half were using air). Circumstantial evidence to support this is the observation that the responses to exercise-training in both groups, although statistically significant, were smaller than expected and did not achieve the minimum clinically important difference of the incremental shuttle walk test nor some domains of the Chronic Respiratory Questionnaire following the exercise training programme.

Another important consideration is that most participants had modest EID, and the trial was therefore underpowered to detect an effect of supplemental oxygen in patients with more severe EID (nadir below $80 \%$ ). Furthermore, prior to the start of the exercise programme, the acute physiological response to oxygen was not tested, hence it is not clear as to the proportion of acute oxygen responders and non-responders in the treatment and control groups. Hence the results of the trial cannot be extrapolated to the specific subgroups of patients with COPD and severe EID, those who have shown a beneficial response to acute oxygen administration, nor patients with non-COPD disorders (such as idiopathic pulmonary fibrosis and pulmonary hypertension) and EID. Further research is required to understand the role of supplemental oxygen in the exercise training of these patient subgroups.

AuIson et al. [15] should be congratulated in rigorously conducting the largest study to date to examine the role of supplemental oxygen during exercise training in patients with COPD and EID. They provide convincing data that the majority of patients with COPD and EID safely benefit from exercise training without the need for supplemental oxygen. This supports the recommendations of international pulmonary rehabilitation guidelines that supplemental oxygen should not be routinely prescribed in patients with COPD who desaturate but do not fulfil indications for long-term oxygen therapy [20-22]. The findings therefore may help to simplify the assessment process of patients with EID for exercise training interventions such as pulmonary rehabilitation. We present our current assessment algorithm prior to commencement of pulmonary rehabilitation (figure 1), which acknowledges remaining uncertainty around the role of supplemental oxygen in specific patient subgroups, namely those who show an acute response to supplemental oxygen and those with severe EID.

Although Alison et al. [15] intended to explore whether exercise training or pulmonary rehabilitation in patients with EID could be enhanced with supplemental oxygen, perhaps the most important clinical implication of this study is that for the vast majority of patients, the non-availability of supplemental oxygen need not be a barrier to exercise training, therefore allowing the option of community and non-medical settings to deliver supervised pulmonary rehabilitation. This potentially increases the accessibility, utilisation and reach of an evidence-based intervention that is considerably more effective in reducing symptom burden in COPD than supplemental oxygen alone.

Conflict of interest: J.A. Walsh has nothing to disclose. M. Maddocks has nothing to disclose. W.D-C. Man reports personal fees from Jazz Pharmaceuticals, Mundipharma and Novartis, grants from Pfizer, National Institute for Health Research and British Lung Foundation, non-financial support from GSK, outside the submitted work.

\section{References}

1 Long term domiciliary oxygen therapy in chronic hypoxic cor pulmonale complicating chronic bronchitis and emphysema. Report of the Medical Research Council Working Party. Lancet 1981; 1: 681-686.

2 Continuous or nocturnal oxygen therapy in hypoxemic chronic obstructive lung disease: a clinical trial. Nocturnal Oxygen Therapy Trial Group. Ann Intern Med 1980; 93: 391-398.

3 Ameer F, Carson KV, Usmani ZA, et al. Ambulatory oxygen for people with chronic obstructive pulmonary disease who are not hypoxaemic at rest. Cochrane Database Syst Rev 2014; 6: CD000238.

4 Chaouat A, Weitzenblum E, Kessler $\mathrm{R}$, et al. A randomized trial of nocturnal oxygen therapy in chronic obstructive pulmonary disease patients. Eur Respir J 1999; 14: 1002-1008.

5 Uronis HE, Ekstrom MP, Currow DC, et al. Oxygen for relief of dyspnoea in people with chronic obstructive pulmonary disease who would not qualify for home oxygen: a systematic review and meta-analysis. Thorax 2015; 70: 492-494.

6 Somfay A, Porszasz J, Lee SM, et al. Dose-response effect of oxygen on hyperinflation and exercise endurance in nonhypoxaemic COPD patients. Eur Respir J 2001; 18: 77-84.

7 O'Donnell DE, Bain DJ, Webb KA. Factors contributing to relief of exertional breathlessness during hyperoxia in chronic airflow limitation. Am J Respir Crit Care Med 1997; 155: 530-535.

8 Cotes JE, Gilson JC. Effect of oxygen on exercise ability in chronic respiratory insufficiency; use of portable apparatus. Lancet 1956; 270: 872-876.

9 Bradley JM, O'Neill B. Short-term ambulatory oxygen for chronic obstructive pulmonary disease. Cochrane Database Syst Rev 2005; 4: CD004356.

10 Marcus CL, Bader D, Stabile MW, et al. Supplemental oxygen and exercise performance in patients with cystic fibrosis with severe pulmonary disease. Chest 1992; 101: 52-57.

11 Garrod R, Paul EA, Wedzicha JA. Supplemental oxygen during pulmonary rehabilitation in patients with COPD with exercise hypoxaemia. Thorax 2000; 55: 539-543. 
12 Rooyackers JM, Dekhuijzen PN, Van Herwaarden CL, et al. Training with supplemental oxygen in patients with COPD and hypoxaemia at peak exercise. Eur Respir J 1997; 10: 1278-1284.

13 Emtner M, Porszasz J, Burns $\mathrm{M}$, et al. Benefits of supplemental oxygen in exercise training in nonhypoxemic chronic obstructive pulmonary disease patients. Am J Respir Crit Care Med 2003; 168: 1034-1042.

14 Spielmanns M, Fuchs-Bergsma C, Winkler A, et al. Effects of oxygen supply during training on subjects with COPD who are normoxemic at rest and during exercise: a blinded randomized controlled trial. Respir Care 2015; 60: 540-548.

15 Alison JA, McKeough ZJ, Leung RWM, et al. Oxygen compared to air during exercise training in COPD with exercise-induced desaturation. Eur Respir J 2019; 53: 1802429.

16 Borg GA. Psychophysical bases of perceived exertion. Med Sci Sports Exerc 1982; 14: 377-381.

17 Nolan CM, Maddocks M, Canavan JL, et al. Pedometer step count targets during pulmonary rehabilitation in chronic obstructive pulmonary disease. A randomized controlled trial. Am J Respir Crit Care Med 2017; 195: $1344-1352$.

18 Curtis KJ, Meyrick VM, Mehta B, et al. Angiotensin-converting enzyme inhibition as an adjunct to pulmonary rehabilitation in chronic obstructive pulmonary disease. Am J Respir Crit Care Med 2016; 194: 1349-1357.

19 Man WD, Soliman MG, Gearing J, et al. Symptoms and quadriceps fatigability after walking and cycling in chronic obstructive pulmonary disease. Am J Respir Crit Care Med 2003; 168: 562-567.

20 Spruit MA, Singh SJ, Garvey C, et al. An Official American Thoracic Society/European Respiratory Society Statement: key concepts and advances in pulmonary rehabilitation. Am J Respir Crit Care Med 2013; 188: e13-e64.

21 Bolton CE, Bevan-Smith EF, Blakey JD, et al. British Thoracic Society guideline on pulmonary rehabilitation in adults: accredited by NICE. Thorax 2013; 68: Suppl. 2, ii1-ii30.

22 Alison JA, McKeough ZJ, Johnston $\mathrm{K}$, et al. Australian and New Zealand pulmonary rehabilitation guidelines. Respirology 2017; 22: 800-819. 